\title{
Activity of Salivary $11 \beta$-Hydroxysteroid Dehydrogenase Type 2 Becomes the Index for the Continuous Strength Exercise to Prevent Locomotive Syndrome in Japan
}

\author{
Noboru Hasegawa1, Maki Ohara², Miyako Mochizuki ${ }^{3}$ \\ ${ }^{1}$ Department of Health and Medical Sciences, Ishikawa Prefectural Nursing University, \\ Kahoku, Japan \\ ${ }^{2}$ Noto-Cho Government Office Health Welfare Section, Hosu-Gun, Japan \\ ${ }^{3}$ Kyoto Bunkyo Junior College, Uji, Japan \\ Email: hsgwn@ishikawa-nu.ac.jp
}

Received 19 September 2015; accepted 26 October 2015; published 29 October 2015

Copyright (C) 2015 by authors and Scientific Research Publishing Inc.

This work is licensed under the Creative Commons Attribution International License (CC BY). http://creativecommons.org/licenses/by/4.0/

(c) (i) Open Access

\begin{abstract}
The Japanese Orthopedic Association proposed a concept called locomotive syndrome (LS) to identify middle-aged and older adults at high risk of requiring health care services because of problems with locomotion-associated lower muscle mass. To prevent LS, it is important to increase muscle mass and muscle strength in middle-age by continuous resistance training. A total of 38 men and women were assessed at baseline and 6 months. Body composition, physical strength and salivary cortisol and cortisone were analyzed. The exercise intervention program was performed by individual muscle endurance level. Body weight, muscle weight and basal metabolism were increased after exercise intervention. The 30-second sit-up test and 3-minute walking were increased, and the 10-time sit-to-stand was decreased significantly. This may be related to increase of leg and abdominal muscular strength. The exercise intervention program increased salivary 11 $\beta$-hydroxysteroid dehydrogenase type 2 (11/-HSD2) activities significantly. These results suggested that $11 \beta$-HDS2 became the index for the increase of muscular strength to prevent LS.
\end{abstract}

\section{Keywords}

Salivary Cortisol, Salivary Cortisone, 11ß-Hydroxysteroid Dehydrogenase, Muscle Weight, Strength Exercise 


\section{Introduction}

In 2015, the average life expectancy of Japanese male and female is 80.5 and 86.8 years, respectively. Japan has the highest proportion of older adults and "Super-aged society" in the world. These results suggest that they are experiencing difficulty in walking, housework and shopping in daily life. These are the main reasons for becoming bedridden or requiring nursing care. The Japanese Orthopedic Association proposed the concept of locomotive syndrome (LS) in 2007 [1] [2]. LS means being restricted in one's ability to walk or lead a normal life owing to a dysfunction in one or more of the parts of the LS (e.g., muscles, bones, joints, cartilage, or the intervertebral discs) [3]. The JOA has prepared a self-check list for this syndrome [4]. It is identified by middle-aged and older adults at high risk of requiring health care services because of problems with locomotion-associated lower skeletal muscle mass. This may be associated with osteoporosis-related fractures, osteoarthritis, spondylosis and sarcopenia. To prevent LS, it is important to increase muscle mass and strength by continuous resistance training in middle age. The focus of the present study was thus middle-aged male/female. Because the quality of appropriate training strength is different among individuals, an index for each person is necessary to continue effectively. Cortisol measurement has been implemented to assess physical stress to exercise [5]. We paid due attention to saliva cortisol having non-invasive and easy-sampling advantages.

Cortisol concentration has emerged as a marker of stress responsibility and a hormonal biomarker of the hypothalamic-pituitary-adrenal axis functioning [6]. Cortisone is derived from the peripheral metabolism of cortisol. In the liver and adipose tissue, $11 \beta$-hydroxysteroid dehydrogenase type 1 (11 $\beta$-HSD1) converts cortisone (inactive form) to cortisol (active form). In the kidney and colon, 11 $\beta$-hydroxysteroid dehydrogenase type 2 (11 $\beta$-HSD2) converts cortisol to cortisone. To corroborate the presence of a glucocorticoid-related stress, it is desirable to consider the cortisol-cortisone shuttle [7].

In human skeletal muscle, $11 \beta$-HSD1 expression was increased in women over 60 years of age and associated with reduced grip strength [8]. Both endurance and strength exercise caused an acute increase of urinary $11 \beta$-HSD1 activity not $11 \beta$-HSD2 activity in healthy trained males [9]. These results suggest that $11 \beta$-HSD1 is the index of endurance exercise.

$11 \beta$-HSD2 is expressed within the salivary glands and the evaluation of salivary $11 \beta$-HSD2 activity is clinically useful to estimate serum-free cortisol because corticosteroid-binding globulin is not present in parotid tissue [10]. The effect of salivary $11 \beta$-HSD2 activity in the effective exercise to prevent muscle weight reduction in healthy sedentary is not known. $11 \beta$-HSD2 activity was estimated by calculating the ratios of the salivary cortisone (SalE)/(SalE + salivary cortisol (SalF)) [11].

Therefore, the present study was designed to investigate the effect of salivary $11 \beta$-HSD2 activity in the effective muscle strengthening exercise with increased muscle weight and basal metabolism to prevent LS by the measurement of SalE and SalF.

\section{Methods}

\subsection{Subjects and Setting}

Prior to the study, approval was obtained from the ethics committee of Ishikawa Prefectural University (project registration number: 468). The study was conducted with sedentary men and women in Noto Peninsula, Ishikawa Prefecture, Japan to prevent locomotive syndrome. After obtaining informed consent, we enrolled 12 Japanese men (age: mean \pm SE $44.1 \pm 11.2$; BMI $23.8 \pm 2.6$ ) and 14 women (age: mean \pm SE $47.9 \pm 8.9$; BMI $23.6 \pm$ 2.1) in this study.

\subsection{Study Design}

A descriptive, comparative pre/post design was utilized. Assessments were undertaken at baseline and 6-month follow-ups. This study was a part of the project carried out in Noto-cho. A six-month exercise classroom is often held by municipalities. Body composition and physical strength were analyzed before and after intervention. Body composition (muscle and fat weight), body weight and basal metabolism were measured using InBody 430 (Biospace Japan, Inc., Tokyo, Japan). The 30-second sit-up test, 10-time sit-to-stand test and 3-minute walking test were assessed. The following 6 exercise menus for increased muscular strength were performed by individual muscle level, composed as follows: 2 or $3 \times 10$ repetitions of bench-press (greater rectoral mucle), leg extension (straight muscle of thigh), leg curl (hamstring muscle), squat (gluteus maximus, quadriceps femoris muscle), 
pull-down exercise (musculus latissimus dorsi) and lateral rise (deltoid muscle) once a week. Therefore, the training menu in this study is all targeted to the training of big muscle.

\subsection{Cortisol and Cortisone Assay}

Saliva samples were centrifuged at $3500 \mathrm{rpm}$ for $10 \mathrm{~min}$ and stored at $-30^{\circ} \mathrm{C}$ for later analysis. On the day of assay, samples were thawed and cortisol was assayed using a enzyme-linked immnosorbent assay kit specifically designed for use with saliva (Salimetrics, USA). Cortisone was assayed using chemiluminescent enzyme immunoassay kit (Arbor Assays, USA).

\subsection{Statistics}

The differences between before and after intervention are evaluated using the paired $t$-test. A probability value of less than 0.05 was conducted to indicate statistical significance.

\section{Results}

\subsection{Exercise Effectiveness in Body Composition}

The mean values of the difference between before and after exercise intervention of body weight, muscle weight and basal metabolism were 1.16 (from -2.5 to 5.5 ) and 0.28 (from -1.1 to 1.9 ) and 10.1 (from -126 to 50), respectively. Body weight was significantly increased $(p<0.01)$ and muscle weight and basal metabolism tended to be increased after exercise intervention for 6 months. Fat weight was not changed (Figure 1).

\subsection{Exercise Effectiveness in Physical Strength}

The mean value of the difference between before and after exercise intervention of 30-second sit-up test, 3minute walking and 10-time sit-to-stand test were 2 (from -3 to 7 ), 15 (from -55 to 105 ) and -1.56 (from -17 to 6.14), respectively. The 30 -second sit-up test $(p<0.01)$ and 3 -minute walking test $(p<0.05)$ were increased, and 10-time sit-to-stand test was decreased $(p<0.05)$ significantly (Figure 2).

\subsection{Exercise Effectiveness in Salivary 11 $\beta$-HSD2 Activity}

The mean value of the difference between before and after exercise intervention was 0.089 (from -0.06 to 0.56 ). $11 \beta$-HSD2 activity was significantly increased $(p<0.05)$ (Figure 3 ).
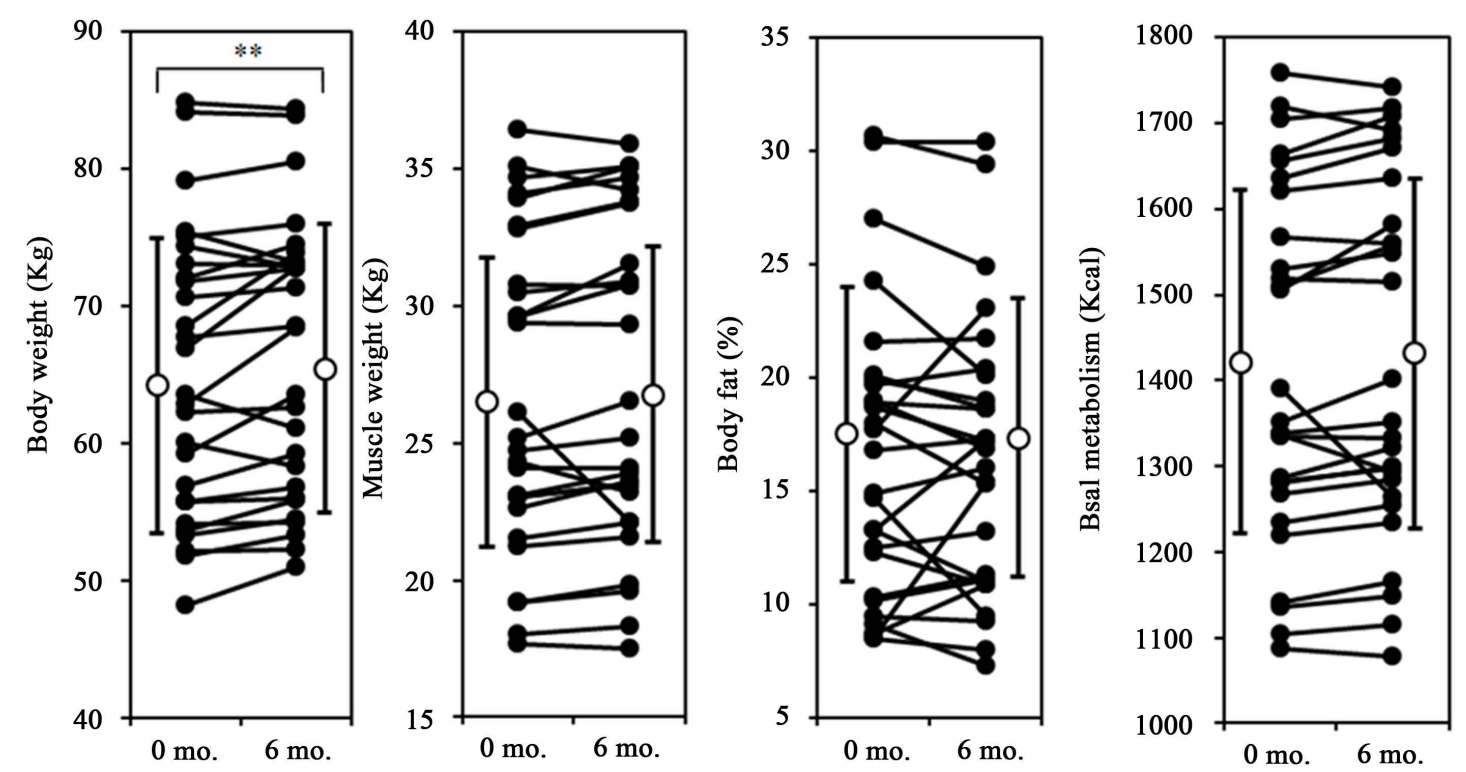

Figure 1. Effects of resistance training on body composition. ${ }^{* *} p<0.01$ as compared with 6 -month. 

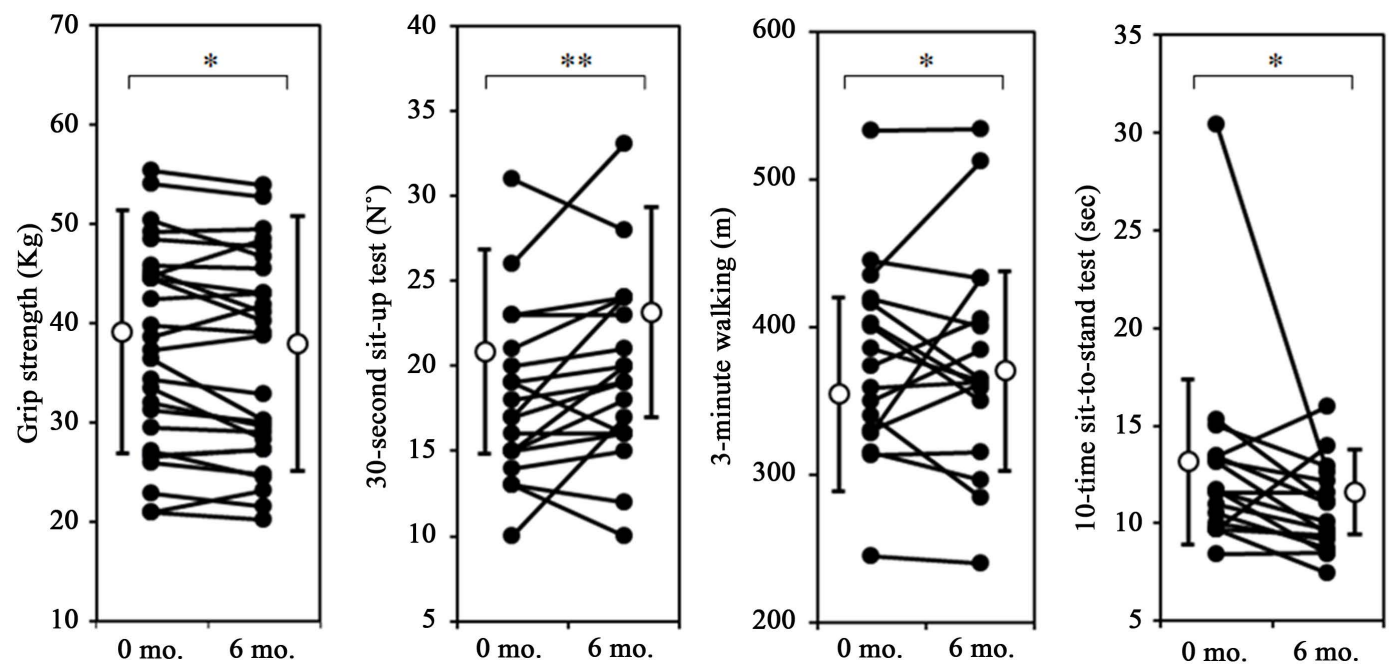

Figure 2. Effects of resistance training on physical strength. ${ }^{*} p<0.05$ as compared with 6 -month; ${ }^{* *} p<0.01$ as compared with 6-month.

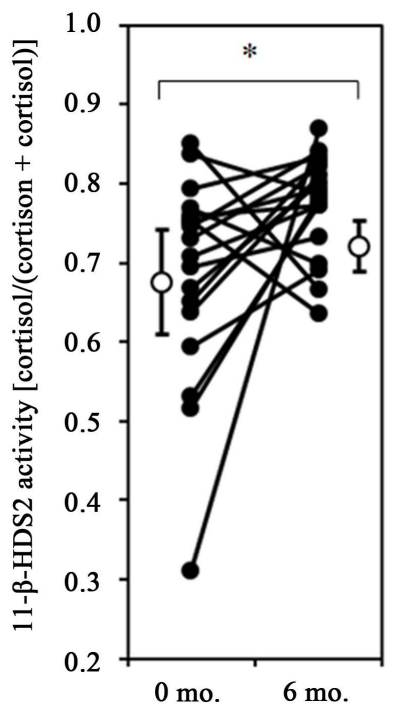

Figure 3. Effects of resistance training on saliva HSD2. ${ }^{*} p<0.05$ as compared with 6 -month; ${ }^{* *} p<0.01$ as compared with 6-month.

\section{Discussion}

In the present study, no difference was observed in fat weight, whereas the increase in muscle weight was found by the exercise support program. These results suggest that this exercise program is the optimal exercise content to be selected in consideration of individual past physical data.

The exercise intervention produced considerable improvement in the leg and abdominal muscular strength (30-second sit-up test and 10-time sit-to-stand test), but not in the whole body endurance (3-minute walking test). The lower limb muscular strength is important to maintain walking functions. In this study, 11 $\beta$-HDS2 expression was associated with increased muscle strength. These results suggested that salivary 11 $\beta$-HDS2 became the index for the increase of muscular strength to prevent LS. Intense exercise increased 11 $\beta$-HSD1 activity and cortisol content [9], where it could contribute to exercise-induced stress. Cortisol is said to be a stress hormone secreted from an adrenal cortex by stress. Blood cortisol is known to be converted into cortisone by $11 \beta$ HDS2, and cortisone is to be converted into cortisol by 11/HDS1 (cortisol-cortisone shuttle) [7]. The cortisol-cortisone shuttle is of importance for the corticosteroid regulation. In previous study, perspectives on the effect of exercise 
on saliva cortisol content have not yet been unified. The exercise increased cortisol [12], decreased cortisol [13] and did not affect cortisol content [14]. The ratio of cortisol and cortisone content must be considered (11/HDS). In this study, stress was decreased by the expression of $11 \beta$-HSD2 and 6-month exercise intervention was maintained.

The main need for care in Japan in 2010 was cerebrovascular diseases followed by dementia, debility, articular diseases and fracture (Ministry of Health, Labour and Welfare, Japan). These findings show that exercise associated with saliva cortisol as an index may be beneficial and should be considered in the overall management of middle-aged and older adults. Moreover, it will be effective as an index of the rehabilitation.

\section{Acknowledgements}

This study was supported in part by the Research Project from Daido Life Welfare Foundation, Japan (2013).

\section{References}

[1] Japanese Orthopedic Association (2010) Guidebook on Locomotive Syndrome. Bunkodo, Tokyo. (In Japanese)

[2] Nakamura, K. (2008) A “Super-Aged” Society and the "Locomotive Syndrome”. Journal of Orthopaedic Science, 13, 1-2.

[3] Japanese Orthopedic Association (2013) Locomotive Syndrome Pamphlet. https://locomo-joa.jp/en/index.pdf

[4] Matsui, Y., Takemura, M., Harada, A., Ando, F. and Shimokata, H. (2013) Utility of "Loco-Check,” Self-Checklist For "Locomotive Syndrome” as a Tool for Estimating the Physical Dysfunction of Elderly People. Health, 5, 97-102.

[5] Gatti, R. and De Palo, E.F. (2011) An Update: Salivary Hormones and Physical Exercise. Scand. Journal of Science and Medicine in Sport, 21, 157-169.

[6] Frodl, T. and O’kaeane, V. (2013) How Does the Brain Deal with Cumulative Stress? A Review with Focus on Developmental Stress, HPA Axis Function and Hippocampal Structure in Human. Neurobiology of Disease, 52, 24-37. http://dx.doi.org/10.1016/j.nbd.2012.03.012

[7] Quinkler, M. and Stewart, P.M. (2003) Hypertension and the Cortisol-Cortisone Shuttle. The Journal of Clinical Endocrinology \& Metabolism, 88, 2384-2392. http://dx.doi.org/10.1210/jc.2003-030138

[8] Hassan-Smith, Z.K., Morgan, S.A., Sherlock, M., Hughes, B., Taylor, A.E, Lavery, G.G, Tomlinson, J.W. and Stewart, P.M. (2015) Gender-Specific Differences in Skeletal Muscle 11 $\beta$-HSD1 Expression across Healthy Aging. The Journal of Clinical Endocrinology \& Metabolism, 100, 2673-2681. http://dx.doi.org/10.1210/jc.2015-1516

[9] Dovio, A., Roveda, E., Sciolla, C., Montaruli, A., Raffaelli, A., Saba, A., Calogiuri, G., De Frabcua, S., Borrione, P., Salvadori, P., Carandente, F. and Angeli, A. (2010) Intense Physical Exercise Increases Systemic 11 $\beta$-Hydroxysteroid Dehydrogenase Type 1 Activity in Healthy Adult Subjects. European Journal of Applied Physiology, 108, 681-687. http://dx.doi.org/10.1007/s00421-009-1265-5

[10] Perogamvros, I., Keevil, B.G., Ray, D.W. and Trainer, P.J. (2010) Salivary Cortisone Is A Potential Biomarker for Serum Free Cortisol. The Journal of Clinical Endocrinology \& Metabolism, 95, 4951-4958. http://dx.doi.org/10.1210/jc.2010-1215

[11] La Marca-Ghaemmaghami, P., La Marca, R., Dainese, S.M., Haller, M., Zimmermann, R. and Ehlert, U. (2013) The Association between Perceived Emotional Support, Maternal Mood, Salivary Cortison, Salivary Cortisone, and the Ratio between the Two Compounds in Response to Acute Stress in Second Trimester Pregnant Women. Journal of Psychosomatic Research, 75, 314-320. http://dx.doi.org/10.1016/j.jpsychores.2013.08.010

[12] Seidman, D.S., Dolev, E., Deuster, P.A., Burstein, R., Arnon, R. and Epstein, Y. (1990) Androgenic Response to LongTerm Physical Training in Male Subjects. International Journal of Sports Medicine, 11, 421-424. http://dx.doi.org/10.1055/s-2007-1024831

[13] Tabata, I., Atomi, Y., Mutoh, Y. and Miyashita, M. (1990) Effect of Physical Training on the Responses of Serum Adrenocorticotropic Hormone during Prolonged Exhausting Exercise. European Journal of Applied Physiology and Occupational Physiology, 61, 188-192. http://dx.doi.org/10.1007/BF00357597

[14] Filaire, E., Duché, P., Lac, G. and Robert, A. (1996) Saliva Cortisol, Physical Exercise and Training: Influences of Swimming and Handball on Cortisol Concentrations in Women. European Journal of Applied Physiology and Occupational Physiology, 74, 274-278. http://dx.doi.org/10.1007/BF00377450 